\title{
Factores que inciden en la innovación organizacional de las Mipymes en México
}

\section{Saul Alfonso Esparza Rodríguez ${ }^{1}$ Universidad Michoacana de San Nicolás de Hidalgo}

\section{Ensayo}

Material original autorizado para su primera publicación en el Journal de Ciencias Sociales, Revista Académica de la Facultad de Ciencias Sociales de la Universidad de Palermo.

Recepción: 21-03-2021

Aceptación: 1-09-2021

Resumen: El presente ensayo presenta una argumentación acerca de la relevancia de los grupos de interés como fuentes internas y externas en el proceso de innovación organizacional. Así pues, se presentan en este trabajo los hallazgos obtenidos en la revisión de la literatura sobre el tema, incluyéndose una serie de conceptos relacionados, así como también referencias que conforman la base de la gestión de grupos de interés para explicar cómo interactúan -directa e indirectamente- con las Mipymes mexicanas en esta forma de actividad innovadora. A su vez, se identifica a los stakeholders (es decir, a los administradores, empleados, clientes, proveedores, competidores, instituciones de crédito y gobierno) como partes interesadas de gran importancia para el proceso de innovación organizacional que se da en las pequeñas y medianas empresas. La conclusión del trabajo permite señalar que las múltiples interacciones entre grupos de interés de las Mipymes mexicanas son factores fundamentales para: en primer lugar, comprender en mejor medida el entorno organizacional interno y externo de estas organizaciones; en segundo lugar, para explicar la introducción de cambios orientados a mejorar el funcionamiento de estas empresas y, finalmente, para dar cuenta de la participación de los administradores dentro de esta dinámica organizacional.

Palabras clave: innovación organizacional; pymes; stakeholders; dinámica empresarial.

\section{Factors that influence the organizational innovation of Mexican SME's}

\footnotetext{
${ }^{1}$ Candidato a Doctor en Administración en la Facultad de Contaduría y Ciencias Administrativas de la Universidad Michoacana de San Nicolás de Hidalgo. Maestro en Administración de Instituciones de Educación Superior por el Instituto Tecnológico y de Estudios Superiores de Monterrey.

Correo electrónico: saul.aer@gmail.com
} 
Abstract: This essay presents an argument about the relevance of the stakeholders as internal and external sources in the organizational innovation process. Thus, the findings obtained in the review of the literature on the subject are presented in this work, including a series of related concepts, as well as references that form the basis of stakeholder management to explain how they interact -directly and directly. indirectly- with Mexican SMEs in this form of innovative activity. At the same time, stakeholders (that is, administrators, employees, clients, suppliers, competitors, credit institutions and government) are identified as concerned parties of great importance for the organizational innovation process that occurs in small and medium-sized businesses. The conclusion of the work allows us to point out that the multiple interactions between interest groups of Mexican SMEs are fundamental factors for: firstly, to better understand the internal and external organizational environment of these organizations; second, to explain the introduction of changes aimed at improving the operation of these companies and, finally, to account for the participation of administrators within this organizational dynamic.

Keywords: organizational innovation, SME's, stakeholders, organizational dynamics.

\section{Introducción}

El presente ensayo presenta un razonamiento orientado a defender la tesis relativa a que los grupos de interés son factores relevantes que inciden en la innovación organizacional de las Micro, pequeñas y medianas empresas (Mipymes) en México. A partir de esto, en el trabajo se ofrece una revisión crítica de la literatura sobre distintos autores que han estudiado el tema, se realiza un análisis sobre la relevancia de las partes interesadas en esta forma de innovación, se plantea una postura argumentativa y se desarrolla la correspondiente defensa en esos términos.

Vale decir que las Mipymes se erigen como las organizaciones con la mayor cantidad de unidades económicas y productivas en el país. La clasificación de estas organizaciones varía según el número de personas empleadas (hasta 250 individuos en el sector industria, y hasta 100 en los sectores comercio y servicios).

En primera instancia, es preciso resaltar la relevancia de las Mipymes en el mundo entero, dada la influencia del sector en aspectos relativos a la economía y empleo a distintos niveles, debido a que es la forma de unidad económica preponderante en prácticamente todas las naciones del planeta. Las estadísticas muestran que, a nivel mundial, representan el $99 \%$ del total de unidades económicas existentes, generando alrededor del $60 \%$ de los empleos y entre el 50 y el $60 \%$ del valor agregado de los países pertenecientes a la Organización para la Cooperación y el Desarrollo Económicos (OCDE, 2019). 
En México, por su parte, la participación de estos organismos en la vida económica y social del país es determinante. A tal punto que ellos forman parte de la cotidianeidad de la vida, pues prácticamente cada día se tiene contacto con alguna empresa como estas para satisfacer algún tipo de necesidad de los consumidores; y es notable que este tipo de unidades económicas se relacionen con procesos de innovación organizacional a partir de la interacción con diversas fuentes, tanto internas como externas, para el desarrollo de nuevos procesos organizativos, nuevas prácticas empresariales y nuevas formas de gestionar las relaciones exteriores de las empresas, que son las dimensiones características de la innovación organizacional (INEGI, 2017).

El Instituto Nacional de Estadística y Geografía de México (INEGI) presenta, en la Encuesta Nacional de Productividad y Competitividad de las Micro, Pequeñas y Medianas Empresas, diversos indicadores relativos a esta naturaleza de actividad innovadora: en el periodo 2016-2017 un total de 169,452 empresas de esta categoría introdujeron algún tipo de innovación organizacional de las cuales, en términos porcentuales, el sector comercio tuvo una mayor participación con el $48 \%$, seguido de los servicios con el $42 \%$, y terminando con la manufactura con el 10\% (INEGI, 2019).

En el mismo orden de ideas, otro instrumento relevante para la medición de la innovación organizacional, denominado Encuesta sobre Innovación y Desarrollo Tecnológico (ESIDET), señala que existen fuentes internas y externas altamente significativas para la realización de actividades orientadas hacia la innovación de tipo organizacional. Como actividades internas se pueden listar: las áreas de investigación y desarrollo tecnológico, ingeniería, producción, mercadotecnia, servicios al cliente, diseño e incluso consideraron la influencia de otras empresas de un mismo grupo empresarial. En lo que corresponde a las fuentes externas, se pueden identificar: las empresas de la competencia, los cliente, consultorías nacionales y extranjeras, proveedores, universidades $u$ otros institutos de educación superior, entre otros (INEGI, 2017).

En virtud de lo anterior, dada la relevancia de la innovación organizacional como una condición previa y necesaria para las innovaciones tecnológicas (Lam, 2009), en especial para enfrentar los efectos negativos causados por la pandemia de la enfermedad COVID19, las posibilidades de supervivencia y adaptación de las empresas mexicanas se fundamenta en la innovación organizacional que otorga beneficios hacia los respectivos stakeholders, integrando enfoques de gestión técnicos, o bien, orientados hacia la sustentabilidad para el cumplimiento de metas y objetivos (Carrillo et al. 2020).

En términos contextuales, la Encuesta sobre el Impacto Económico Generado por COVID-19 en las Empresas (ECOVID- IE), realizada por INEGI, muestra que el $73 \%$ de las empresas instrumentó operaciones para hacer frente a la contingencia, mientras que lo 
mismo hicieron el $59 \%$ de las microempresas. De las empresas medianas y pequeñas, el $51 \%$ tuvo que cerrar de manera temporal o recurrir al paro técnico, mientras que el $60 \%$ de las microempresas tomó las mismas medidas (Organización Internacional del Trabajo - OIT, 2020).

Es en este sentido que se discute si los grupos de interés son fuentes internas y externas de innovación organizacional, para lo cual la estructura argumental que sigue presenta los hallazgos de la revisión de la literatura relativa al tema. Se incluyen una serie de conceptos relacionados con esta forma de innovación, se revisan trabajos que relacionan la gestión de grupos de interés con la innovación organizacional como una base literaria que, seguidamente, permitirá explicar la forma en que ellas interactúan - directa e indirectamentecon las Mipymes mexicanas dentro de este tipo de innovación.

Para finalizar, la conclusión del presente ensayo afirma que las múltiples interacciones entre grupos de interés de las Mipymes mexicanas son factores fundamentales que permiten comprender, en mejor medida, el entorno organizacional tanto a nivel interno como externo. Al mismo tiempo, permite explicar la introducción de cambios orientados a mejorar el funcionamiento de estas empresas y, por último, permite explicar la participación fundamental del administrador dentro de la dinámica organizacional.

\section{Argumentación teórica}

\subsection{La innovación de tipo organizacional}

Con objeto de esclarecer el concepto que se toma como propuesta dentro de este marco argumentativo, en términos generales, se puede decir que la innovación organizacional comprende el desarrollo y la implementación de nuevas ideas por parte de personas que se involucran en transacciones dentro de un orden institucional basado en cinco factores básicos: ideas, resultados, personas, transacciones y conceptos (Arraut, 2008). Estos factores representan guías para comprender aquellos problemas que confrontan los administradores relativos a cuestiones tales como la gestión de la atención, la gestión de nuevas ideas y su aplicación, además de presentar retos en términos de relaciones institucionales y de estrategia orientada al liderazgo institucional (Van de Ven, 2016).

De esta manera, Damanpour (2020) propone que el concepto innovación organizacional comprenda procesos de naturaleza no tecnológica, principalmente en empresas del sector manufacturero, a partir de lo cual, el aprovechamiento de ideas nuevas y su consecuente influencia en el funcionamiento de la organización se hace presente mediante un proceso en el que la creatividad resulta un punto de partida hacia el desarrollo de la organización. En esto suelen coincidir diversas concepciones que explican a la 
innovación en las organizaciones como una actividad que se construye con base en ideas creativas, y que su consecuente implementación se asocia a nuevos productos, procesos o servicios, de acuerdo con la línea de negocio de cada organización. Aquí se pueden considerar, también, nuevos procedimientos o nuevas políticas para la organización en sí misma (Amabile, 1988).

Un aspecto sumamente interesante que se aprecia en la literatura, es el referido a las múltiples interacciones que ocurren hacia dentro de las empresas, entre administradores y empleados; ellas también son influidas por el cambio en el entorno de las organizaciones, son representadas por un mundo en constante cambio al cual se suele reaccionar mediante una utilización de diversas innovaciones organizacionales tales como: la implementación de técnicas relativas a la división estratégica del trabajo, la gestión de la calidad total, el sistema de producción Toyota y la producción esbelta (Steiber y Alänge, 2015).

Por ello, dado que un organismo social siempre contará con la posibilidad de mejorar para generar mejores resultados, todas las funciones pueden ser objeto de una actividad de innovación, como lo menciona la versión actualizada del Manual de Oslo, en donde se consideran las funciones básicas del negocio para la producción de bienes y servicios, así como también las funciones de soporte: distribución, logística, marketing, ventas y servicios posventa; los servicios tecnologías de la información y la comunicación (TICs) para la empresa; o el proceso de desarrollo del negocio y de los productos (OCDE y UE, 2018).

Por lo anteriormente mencionado, y teniendo en cuenta la relevancia de factores internos, tales como la interacción entre administradores y empleados, o bien de los factores externos del entorno empresarial (relativos a la influencia de proveedores, competidores, clientes, instituciones de crédito y gobierno), es posible argumentar que este fenómeno social se genera mediante la aplicación de cambios hacia dentro de la organización (en la cultura, estructura, procesos y/o sistemas), en su relación con el mercado y las redes de negocio (que incluyen la base de clientes, la relación con los proveedores, la reputación), o sobre los productos de la organización (los bienes y servicios propiamente dichos) (Ricciardi, 2014).

\subsection{Factores que inciden en la innovación organizacional de las Mipymes} mexicanas: los grupos de interés

De acuerdo con la literatura, los grupos de interés (stakeholders) son aquellas personas $u$ organizaciones que son afectadas, pueden afectar o pueden percibirse afectadas debido al desarrollo de las actividades productivas de una empresa (Freeman, 2004). En ese sentido, el enfoque de gestión de expectativas y necesidades de los stakeholders enfatiza la necesidad de comprender la interconexión de las relaciones entre empleados, clientes, proveedores, inversionistas, comunidades y cualquier persona que tenga algún interés en el desarrollo de la organización (Freeman, 2017). 
Con el objetivo de explicar la relación entre grupos de interés y la innovación organizacional en las Mipymes mexicanas, vale revisar la información provista por la encuesta sobre innovación y desarrollo tecnológico (ESIDET) realizada por INEGI (2017), en donde las fuentes internas y externas de innovación se aprecian relevantes para la implementación de innovaciones de tipo organizacional, las cuales, a su vez, puede ser trazadas hacia algún tipo de grupo de interés (interno, externo, actual o potencial).

Las organizaciones atraviesan el proceso orientado al desarrollo, producción, distribución, o adopción (mediante la selección, implementación y monitoreo) de innovaciones (Damanpour, 2020), gracias a un proceso de intercambio entre sus respectivos grupos de interés. De esta manera, lo que un stakeholder contribuye servirá como una recompensa o delimitación para otro en un proceso dinámico en donde se busca mantener un balance organizacional (Adler et al. 2004); y, en ese sentido, la fricción entre las partes interesadas se convierte en una fuente de innovación organizacional.

Por ello, se procede - a continuación- a analizar la literatura disponible acerca de los grupos de interés particulares, es decir, a revisar la forma en la cual administradores, empleados, clientes, proveedores, competidores, instituciones de crédito y gobiernos influyen en la innovación organizacional de las empresas, y a apreciar la interacción dinámica que existe entre estas partes interesadas y el proceso de implementación de cambios orientado a mejorar el desempeño de las organizaciones a partir de nuevos métodos organizativos, nuevas prácticas empresariales y/o nuevas formas de gestionar las relaciones exteriores de las empresas.

\subsubsection{Administradores}

Los administradores representan el punto de convergencia entre las tensiones, el grado de influencia y el cumplimiento de expectativas de las partes interesadas de la organización, pero también son un grupo de interés en sí mismos dada la necesidad de obtener resultados que generalmente se orientan a lograr el mayor beneficio con el mínimo de recursos en la coordinación de los elementos con los que se conforma un organismo social.

En ese sentido, hay que destacar que las estrategias de innovación tienen un efecto positivo en la capacidad de las organizaciones para resolver tensiones entre diversos objetivos organizacionales, lo cual puede lograrse mediante el uso de recursos y capacidades para implementar estrategias de innovación inimitables, fomentando el logro de ventajas competitivas mediante la implementación de prácticas relacionadas con la innovación abierta para obtener recursos y competencias creadas por actores externos en una forma rápida y eficiente (Silva et al. (2021). 
La figura del administrador tiene un rol clave en el desarrollo de las ideas novedosas y creativas que surgen a partir de la interacción entre grupos de interés. De tal manera que la forma en cómo la organización adopta innovaciones de tipo organizacional puede trazarse como un proceso evolutivo, es decir, desde una primera propuesta hasta la aplicación y evaluación del resultado; este proceso de ajuste, tal y como fue estudiado por Zhuang (2005), podría facilitar la innovación e incrementar el desempeño de la compañía.

Para concluir con este apartado sobre la función de los administradores como punto de convergencia en la gestión de los elementos necesarios para dar respuesta a las expectativas y necesidades, resulta importante revisar cómo se implementan cambios en la coordinación que llevan a cabo los administradores tanto dentro de la organización como más allá de sus límites, en vistas de ubicar y aprovechar los recursos con los que cuenta la organización (Kraśnicka et al. 2017; Watson et al. 2018; Petiz y Mendes Mónico, 2018).

De acuerdo con estudios como los realizados por el Global Entrepreneurship Monitor (GEM) en el ámbito empresarial mexicano (Rodríguez Valencia y Olivares Contreras, 2019), la relevancia de la influencia de la innovación se ha centrado fundamentalmente en el crecimiento económico y en el ámbito de la innovación empresarial, con un papel preponderante de los empresarios y administradores. Ellos, evidentemente, aparecen como los encargados de gestionar y administrar las organizaciones,

\subsubsection{Empleados}

El factor humano en las empresas representa una fuente de creatividad y propuestas para mejorar los procesos organizacionales que se manifiestan tanto en forma de nuevas ideas, como de exigencias y protestas de mejora de las condiciones de trabajo. Este aspecto es claramente referido por Leovaridis y Popescu (2015), quienes describen la interacción del personal en las empresas como un tipo de participación directa o indirecta en el proceso de toma de decisiones. La parte interesada tiene una participación fundamental en los procesos que conllevan a la innovación organizacional, debido a que se relacionan directamente con los procesos de la organización, es decir, representan lo que se ha referido como "dueños del proceso" dentro del área en la que se desempeñan. En ese sentido, es interesante conocer el argumento que presentan investigadores como Van de Ven (2016) acerca de que los fundamentos de la innovación son: las ideas, por un lado, y son los empleados que las desarrollan, implementan, reaccionan y modifican, por otro.

De esta manera, una empresa puede ser tan innovadora como el involucramiento del personal con los procesos mediante los cuales se gestiona la producción de bienes en una empresa, en donde factores tales como el diálogo abierto, el conocimiento compartido, la experimentación desplegada y el aprendizaje adquirido dan forma a la creación de nuevos modelos de colaboración y nuevas relaciones sociales (Totterdill, 2015; Voica et al. 2016). En 
términos del contexto de pequeñas y medianas empresas mexicanas, distintos trabajos han identificado la relevancia de los miembros las organizaciones que poseen, comparten y ejercen una serie de valores cuyo fin último es la satisfacción de las necesidades del cliente, facilitando con ello el logro de una cultura de calidad mediante el cambio y el desarrollo de una serie determinada de valores y actitudes (Ascúa et al. 2015).

\subsubsection{Clientes}

Los clientes representan la auténtica razón de ser de las organizaciones comerciales. Las necesidades de este grupo de interés guían las características de un determinado producto o servicio, el tiempo de entrega, el precio, el ordenamiento productivo, y la participación de la empresa en la industria. Por ello, tal y como proponen Lindblad y Karrbom Gustavsson (2021), mediante la especificación de requerimientos acerca de sus respectivas necesidades, los clientes hacen valer su voz dentro de los procesos organizacionales, siendo satisfechos estos requerimientos mediante la implementación de una red de actores, recursos y actividades de las empresas.

El enfoque productivo de las empresas, en términos de una red de actores interactuando activamente para satisfacer las necesidades de clientes -mientras cumple con las expectativas de otras partes interesadas- permite comprender el grado de complejidad de la situación empresarial, un aspecto que -de acuerdo con Tang et al. (2013)- se basa en la conexión, integración y reducción de conflictos internos en pos de focalizarse en los clientes, mejorar el vínculo con ellos, y, de esta forma, hacer uso del conocimiento que se genere sobre esta parte interesada (Kruse, 2013). La dinámica organizacional es cambiante y compleja debido, precisamente, a las necesidades cambiantes de los clientes, sumado a la presión competitiva extensiva y el cambio tecnológico rápido (AL Ajlouni, 2013). La orientación estratégica con eje en los clientes motiva los comportamientos apropiados de las firmas para que, no sólo se enfoquen en crear y ofrecer un valor superior al cliente, sino también para a que se esfuerce por adoptar una cultura orientada a las competencias de innovación (Racela, 2014).

Con motivo de interpretar lo anteriormente expuesto en términos de la realidad empresarial mexicana, la gestión de la innovación propuesta por autores como López, Blanco y Guerra (2010, citados por Díaz Ramos et al. 2019), exhibe un proceso de gestión de la innovación en donde las variables relevantes se manifiestan a partir de factores intrínsecamente relacionados con los clientes; tales como: la orientación al mercado, la investigación y el desarrollo, el diseño de productos y la creatividad, entre otros aspectos relevantes.

\subsubsection{Proveedores}


Como grupo de interés, los proveedores forman parte de la red de socios externos de la empresa. Son actores que contribuyen al proceso productivo mediante su conexión en términos de creación de valor y cadena de suministro. He et al. (2021) aseguran que la influencia de los proveedores se ve representada en la conexión de este grupo de interés con el proceso de producción, que implica un proceso de aprendizajes externos virtuosos en torno a herramientas administrativas o producción esbelta, y que conlleva un impacto directo sobre la productividad, la ventaja competitiva, las relaciones entre usuarios, el contenido del trabajo, etc. (Alänge et al. 2007).

En términos de la cadena de suministro, los proveedores influyen en la generación de valor dentro de la estructura de trabajo de la organización. Generan procesos de mejora continua a partir de elementos tales como: el intercambio de información, los ajustes en el ciclo de producción (orientados a satisfacer necesidades de clientes), o, incluso, desarrollando nuevas técnicas de gestión en la cadena de suministro en sí misma. Se convierten, así, en un activo intangible que puede llevar a la diferenciación de la empresa y, por ende, a un mayor grado de competitividad (Armbruster et al. 2008; Townsend, 2010; Korhonen y Kaarela, 2011; Mothe y Nguyen-thi, 2011; Morrar et al. 2013).

En lo correspondiente a las empresas mexicanas, las actividades organizacionales innovadoras permiten comprender la importancia que conlleva para las empresas pertenecer a una integración formateada como cadena productiva. Esto ha facilitado a las organizaciones identificarse como entes sociales que generan beneficios a la sociedad mediante la colaboración cercana con proveedores, intermediarios, clientes, gobierno y competidores; generando, en dicho proceso, un modelo donde los participantes comparten beneficios para el mutuo desarrollo económico y social (Vega, 2016).

\subsubsection{Competidores}

Como grupo de interés, los competidores de una industria fomentan la innovación organizacional a través de la interacción de las empresas en el mercado. Ellos utilizan diversos métodos para satisfacer las demandas dinámicas de los clientes a partir de la implementación de formas de gestión que permitan mantener ventajas sobre otros participantes de la industria (van de Vrande et al. 2009). Estas formas de gestión tienden a satisfacer necesidades y a generar ofertas que mejoren las propuestas por otros competidores (Han et al.1998), al tiempo que los ambientes complejos y el clima de incertidumbre, representan un factor definitivamente relevante para la implementación de nuevos procesos de mejora (Siguaw et al. 2006).

Lo anterior se logra a partir de la capacidad de las organizaciones para responder a los cambios del entorno competitivo en donde las empresas generan ofertas de valor para el mercado mediante la satisfacción de necesidades reales o percibidas. Es en ese entorno 
competitivo en donde, según Edison et al. (2010), resulta muy importante conocer la oferta de bienes y servicios de los competidores (reparando sobre cuáles son sus características superiores), en vistas de poder ofrecer a los consumidores nuevas propuestas enriquecidas, que resulten de mayor preferencia (Bigliardi et al. 2011).

Esta relación dinámica con los competidores como grupo de interés puede darse en forma planeada y estratégica, o a manera de reacción forzada frente a las acciones de la competencia en una industria altamente competitiva. Estas dinámicas han sido estudiadas, oportunamente, por Leal-Rodriguez y Albort-Morant (2016), en términos de las habilidades que disponen las organizaciones para renovarse continuamente. Margir et al. (2014), en tanto, pone énfasis en el ciclo evolutivo de los cambios organizacionales de la competencia; mientras que Ejdys (2015) propone que estas actividades sean deliberadamente realizadas.

En este orden de ideas, Larios-Gomez (2016) menciona que, dentro del contexto mexicano, las interacciones entre los competidores y el entorno empresarial presentan retos para ser integrados a las acciones y actividades cotidianas de la organización; sin embargo, asegura que son altamente relevantes en términos de los nuevos paradigmas tecnoeconómicos que están generando una auténtica dependencia de los procesos de crecimiento y desarrollo económico a las capacidades para crear valor mediante la incorporación del conocimiento. La innovación y difusión del uso productivo de la tecnología asumen un rol clave en el objetivo específico de mantener un grado de competitividad en la industria, tal como se ha descrito en los trabajos publicados por la Comisión Económica para América Latina y el Caribe (CEPAL) (Dini y Stumpo, 2019).

\subsubsection{Instituciones de crédito}

Para las empresas, el acceso a fuentes de financiamiento representa un factor de viabilidad empresarial, inversión en factores productivos, mantenimiento o renovación de la capacidad instalada, escalamiento de la producción, o -inclusive- una forma de financiar la nómina de la empresa. Dado este grado de relevancia en los procesos empresariales, las instituciones de crédito son también stakeholders que se involucran en la generación de valor conjunto, y que participan en la implementación de innovaciones (sobre todo de las de tipo administrativas), al facilitar a las Mipymes la implementación de nuevos canales de comunicación y al permitir el aprovechamiento tanto de las plataformas tecnológicas como del conocimiento adquirido durante el proceso relacional (Zhao et al. 2019).

Esta interacción afecta en forma relevante a la relación de la empresa con otros stakeholders, como por ejemplo proveedores (pues el financiamiento permitirá mantener o ampliar la relación con estos grupos de interés, diversificar las compras empresariales o incrementar factores de producción como maquinaria y equipo), competidores (la inversión realizada podría fortalecer el posicionamiento competitivo de la empresa) y también clientes 
(mayores recursos financieros pueden traducirse en mayor producción, ampliación de la línea de productos o mayor calidad en los bienes producidos). Lo anterior sucede debido a que la disponibilidad de capital financiero está relacionada con las actividades tanto de innovación como de investigación y desarrollo. Abouzeedan (2011) y Hámori y Szabó (2011) señalan que las dificultades financieras se mantienen como uno de los factores que más afectan a las empresas pequeñas.

Por lo anteriormente mencionado, situaciones referentes a las distintas interacciones entre grupos de interés, tales como un capital humano adecuadamente entrenado o contar con capacidad financiera para la inversión en investigación y desarrollo (según Barrera Rodríguez et al. (2017), representan factores críticos para alcanzar el éxito. El acceso a recursos financieros, por tanto, es clave para el desarrollo de los pequeños negocios (Ibrahim, 2017); las interacciones entre stakeholders representan un factor de alta relevancia para aquellos administradores que buscan implementar una coordinación eficiente en sus respectivos organismos sociales.

El financiamiento para las Mipyme mexicanas aún representa un tema pendiente en términos de inclusión financiera de estas unidades económicas (las empresas que acceden a estos recursos reportan que su principal fuente es el sistema financiero formal, siguiendo el crédito de proveedores y los recursos propios); sin embargo, esta realidad requiere la implementación de actividades de innovación organizacional que den paso al desarrollo de una planeación adecuada desde donde: formalizar un plan de negocios, analizar el mercado, desplegar estudios financieros y diseñar nuevos modelos de producción; acciones todas ellas que, posiblemente, influyan en el incremento de la productividad (Díaz Ramos et al. 2019).

\subsubsection{Gobierno}

Las instituciones de gobierno son factores fundamentales en el desarrollo de las Mipymes debido a su contribución al fortalecimiento de estas organizaciones. El pago de impuestos, el acceso a financiamiento, la obtención de permisos de operación, los programas de capacitación, o incluso de integración a cadenas de suministro, son algunos ejemplos de estos factores.

Dichas situaciones son referidas por Zhao et al. (2019) como aspectos fundamentales que influyen en las organizaciones y en los individuos para lograr innovación. La estructuración de sistemas de innovación de orden nacional, dentro de la política industrial, de investigación y económica (Bikfalvi, 2007); junto a la colaboración en materia de investigación que puede entretejerse entre universidades e industrias; sumado a las compras de productos tecnológicos avanzados que lleva a cabo el gobierno (Gul y Nouman, 2009), las tres dimensiones reflejan un papel clave en los indicadores de innovación, a tal punto que pueden generar compañías de amplio alcance como política de gobierno (Olson et al. 2011). 
Gracias a las iniciativas de gobierno se ha logrado el fomento de la innovación en las Pymes (Baregheh et al. 2014).

Mediante la revisión de la literatura correspondiente, se identificaron tres elementos institucionales cruciales para estimular el desempeño innovador en la industria: la regulación, los incentivos y la política de gobierno (Sahrom et al. 2016). Asimismo, tanto universidades, centros de investigación y repositorios del conocimiento (Lima et al. 2017), por un lado, como colectivos sociales representados por las agencias regulatorias y de desarrollo económico, por otro, (cada uno con su propio conjunto de normas, expectativas y convenciones acerca de hacer negocios) (Couchman y Beckett, 2013), resultan relevantes para la adopción de nuevos métodos organizativos, nuevas prácticas empresariales y nuevas formas de gestionar las relaciones exteriores de las empresas.

En lo que respecta a la influencia de este grupo de interés como promotor y agente relevante en el desarrollo de las actividades de las empresas, vale mencionar, a continuación, los principales programas y políticas relacionados con financiamiento en México para apoyar a las Mipymes en el marco de la crisis generada por la COVID19: criterios contables especiales aplicables a instituciones de crédito, créditos al consumo, de vivienda y comerciales, para los clientes cuya fuente de pago se encuentre afectada por esta contingencia, programa de microcréditos orientado a municipios o localidades de media, alta o muy alta marginación y/o con altos índices de violencia, crédito orientado a pequeñas y medianas empresas que no hayan despedido trabajadores durante la emergencia, financiamiento a empresas hoteleras, agencias de viajes y servicios de transporte público a tasas preferenciales, y una alternativa de financiamiento a tasas atractivas (OIT, 2020).

Con lo anteriormente argumentado, se detalla la interacción entre las partes interesadas que dan origen a la innovación organizacional en las empresas mexicanas. Seguidamente, se propone una incidencia doble en el proceso de gestión de una empresa: tanto directa como indirecta.

\section{Interacción entre las partes interesadas para lograr la innovación} organizacional

La innovación organizacional puede entenderse como un proceso de gestión de grupos de interés de forma secuencial y simultánea, en donde un producto o servicio representa una suerte de interpretación que realiza el administrador sobre la coordinación de los elementos internos y externos que conforman un organismo social para cumplir con un grado de acuerdo temporal en la dinámica de intercambio que se da entre las partes interesadas en el marco de una organización. Se erige, además, como el resultado de las 
condiciones que rigen las conductas, decisiones, acciones e involucramiento de los stakeholders, es decir, de la influencia, el poder, la legitimidad o de su importancia.

Por ello, la base de la propuesta del presente trabajo es considerar la gestión de grupos de interés como un elemento esencial, en base a resultados, para fomentar un estado de resiliencia organizacional en las empresas, que contribuya a mejorar las posibilidades de supervivencia y/o permanencia en una época histórica signada por las turbulencias y la incertidumbre de naturaleza sanitaria, económica y social.

Los "beneficios compartidos" de una organización con sus respectivos grupos de interés, ubican a la perspectiva organizacional en el centro de la comprensión de las diversas interacciones que se dan entre compañías e individuos (tanto internos como externos), identificando los factores que afectan a la organización, facilitando la interpretación de necesidades convergentes y divergentes que sirven como guías para atender simultáneamente- un amplio espectro de expectativas (Esparza y Martínez, 2019).

Se puede argumentar, entonces, que existe una relación virtuosa por la cual: algún grupo de interés hace uso de la voz de la empresa; la empresa depende de dichos grupos de interés; la parte interesada tiene cierto poder sobre la empresa; el grupo de interés es dependiente de la empresa; la relación entre la empresa y la parte interesada es legítima; y existe una obligación moral de la empresa hacia el grupo de interés (tal y como ha sido propuesto en la importante investigación seminal de Mitchell et al. 1997 sobre la gestión de las partes interesadas).

En definitiva, para ser y mantenerse competitivo en cualquier industria resulta esencial gestionar los recursos de las empresas para que se sostengan en armonía; y en donde el administrador pueda contar con información objetiva proveniente de las principales partes interesadas, pues serán ellas quienes proveerán un enfoque estratégico para tomar las decisiones cruciales que sean necesarias para lograr los objetivos de la forma más eficiente posible.

\section{Conclusiones}

Como se ha argumentado en este ensayo, la influencia de los grupos de interés en la innovación de tipo organizacional que se refleja en las Mipymes mexicanas se manifiesta como un factor fundamental de las dinámicas del entorno -interno y externo- en el cual transitan este tipo de unidades económicas, situación esta que permite comprender la participación básica de la persona encargada de la administración empresarial.

La revisión de la literatura muestra que la función del administrador, en tanto actor determinante en la innovación, se basa en el diseño, implementación, evaluación y 
seguimiento de sistemas, métodos, técnicas, procedimientos y modelos orientados a la coordinación eficiente de los componentes que conforman un organismo social, con el objeto de responder a las distintas necesidades y expectativas de los grupos de interés. E administrador logra esto mediante innovaciones que se suceden gracias trabajo coordinado entre elementos tangibles e intangibles, internos y externos, así como de naturaleza económica y no económica relativas al contexto de las empresas.

A partir del juego entre las partes interesadas, la innovación de tipo organizacional se realiza en etapas: la primera y más importante (aunque no suficiente), consiste en la demostración de una idea basada en propuestas para la implementación de nuevas prácticas empresariales, nuevos métodos organizaciones en el lugar de trabajo y/o nuevas formas de gestión de las relaciones con el exterior de la empresa. La transición hacia la innovación debe llevar a la condición suficiente para que exista una innovación en términos administrativos, esto es, enfocada en la aplicación de una invención organizacional que logre influencia en la participación de la empresa dentro del mercado (o de la industria), que se base en el diseño, implementación, evaluación y generación de procesos orientados al aprovechamiento de los recursos con los que cuenta un organismo social.

Con lo dicho, entonces, vale recalcar que la función del administrador se orienta al diseño de sistemas de gestión que faciliten el tránsito de una invención hacia una innovación, mediante la interacción con grupos de interés internos (como por ejemplo empleados y administradores), y externos (por ejemplo: clientes, proveedores, competidores), incluso con grupos externos pero potenciales (como pueden ser: instituciones de crédito o gobiernos). Los procesos de gestión administrativa, por tanto, deben atender y satisfacer las necesidades de los diversos grupos de interés, tanto en forma simultánea como secuencial, tomando conciencia de que los cambios implementados desde la administración para responder a las condiciones del entorno van a modificar la estructura de la empresa en función del uso y el destino de los recursos organizacionales; razón por la cual un "estado de equilibrio" en la organización se definirá en términos del poder, influencia y legitimidad de cada grupo de interés, en forma ponderada y de acuerdo a la contingencia que enfrenta el organismo social en un momento determinado. En otras palabras, la relevancia de un grupo de interés se determinará en función de la cantidad de recursos destinados a cumplir con sus expectativas particulares que rivalizan con las de las demás partes interesadas, pero dentro de una referencia delimitada por las condiciones del ambiente interno y externo de la organización, en este caso la Mipyme mexicana.

En lo que corresponde a la interacción de las partes interesadas como factores que influyen en la innovación organizacional de las empresas mexicanas, tanto las evidencias documentadas como los trabajos de investigación antes citados exhiben un panorama en el 
cual la relación de las empresas con los grupos de interés internos y externos, junto a los externos e indirectos (como las instituciones de crédito y el gobierno), se manifiesta muy claramente y de forma intensa, sobre todo en un escenario contemporáneo signado por la incertidumbre económica, social, sanitaria y productiva.

\section{Referencias bibliográficas}

Abouzeedan, A. (2011). SME Performance and Its Relationship to Innovation (Disertation 1364). https://www.diva-portal.org/smash/get/diva2:447516/FULLTEXT01.pdf

Adler, N. Shani, A. B. y Styhre, A. (2004). Collaborative Research in Organizations:

Foundations for Learning, Change, and Theoretical Development. Sage.

AL Ajlouni, M. I. (2013). Investigating the impact of knowledge management on organizational innovation: conceptual framework. International Journal of Academic Research, 5(1), 114-119. https://doi.org/10.7813/2075-4124.2013/5$1 / a .18$

Alänge, S., Jacobsson, S. y Jaryehammar, A. (2007). Some aspects of an analytical framework for studying the diffusion of organizational innovations. Technology Analysis \& Strategic Management, 10(1), 3-22. https://doi.org/10.1080/09537329808524301

Amabile, T. (1988). A model of creativity and innovation in organizations. En L. L Cummins y B. M. Staw (Edts), Research in Organizational Behavior (pp. 123-167) Vol. 10. JAI Press.

Armbruster, H., Bikfalvi, A., Kinkel, S. y Lay, G. (2008). Organizational innovation: The challenge of measuring non-technical innovation in large-scale surveys. Technovation, 28(10), 644-657. https://doi.org/10.1016/j.technovation.2008.03.003

Arraut, L. (2008). La innovación de tipo organizacional en las empresas manufactureras de Cartagena de Indias. Semestre Económico, 11(22), 185-203.

Ascúa, R., Roitter, S., Vigier, H., Aggio, C., Baruj, G. y Pavlicevic, J. (2015). Lecturas seleccionadas de la XX Reunión Anual Red Pymes Mercosur.

Baregheh, A., Hemsworth, D. y Rowley, J. (2014). Towards an integrative view of innovation in food sector SMEs. International Journal of Entrepreneurship and Innovation, 15(3), 147-158. https://doi.org/10.5367/ijei.2014.0152

Barrera Rodríguez, A., Cuevas Reyes, V., Ramirez García, A. G. y Espejel García, A. (2017). Identificación de componentes de innovación organizacional para la 
consolidación de empresas asociativas rurales. Revista de Geografía Agrícola, (59), 127-144. https://doi.org/10.5154/r.rga.2017.59.006

Bigliardi, B., Colacino, P. e Ivo, A. (2011). Innovative Characteristics of Small and Medium Enterprises. Journal of Technology Management \& Innovation, 6(2), 83-93. https://doi.org/10.4067/S0718-27242011000200006

Bikfalvi, A. (2007). Innovation, Entrepreneurship and outsourcing : essays on the use of knowledge in business environments. Doctor Europeus (PhD Programme) https://www.tdx.cat/bitstream/handle/10803/7958/tab.pdf?sequence=3\&isAllowe $\mathrm{d}=\mathrm{y}$

Carrillo, D. O. L., Martínez, D. G. R., Sánchez, D. Z. M. C., Real, D. O. B. R., Estrada, D. C. M. C., Ovilla, M. M. Á. G. y Estrada, D. L. de J. V. (2020). Gestión de Mipymes mexicanas en tiempos de COVID-19. Grupo Editorial Hess.

https://ceune.unach.mx/images/Libros_congresos/Gestin_de_Mipymes_mexican as_en_tiempos_de_COVID-19.pdf\#page=291

Couchman, P. K. y Beckett, R. (2013). Construing Innovation as a Social Act: Any Clues for SMEs? British Academy of Management Conference, 21. http://www.bam.ac.uk/ Damanpour, F. (2020). Organizational Innovation: Theory, Research, and Direction. Edward Elgar Publishing

Díaz Ramos, M. A., Melgarejo Galindo, L., Melgarejo Galindo, D., Olivera Gómez, D. A., Vázquez Luna, M., Canul Dzul, J. M., Salgado Morales, E. y Cecilia Ramón Vila, M. D. J. (2019). Innovación tecnológica en las pymes 1. Red Iberoamericana de Academias de Investigación. http://redibai-myd.org/portal/wpcontent/uploads/2020/01/8617-49-4.pdf

Dini, M. y Stumpo, G. (coords). (2019). Mipymes en América Latina: un frágil desempeño y nuevos desafíos para las políticas de fomento. Síntesis. Comisión Económica para América Latina y el Caribe (CEPAL).

Edison, H., bin Ali, N. y Torkar, R. T. (2010). Towards innovation measurement in software industry. Journal of Systems and Software, 86(5), 1390-1407. https://doi.org/10.1016/j.jss.2013.01.013

Ejdys, J. (2015). Market orientation vs. inovativeness of SMEs of Podlaskie province. Verslas: Teorija Ir Praktika, 16(4), 353-361. https://doi.org/10.3846/btp.2015.563

Esparza, S. y Martínez, J. (2019). The shared benefit approach to competitiveness.

Mercados y Negocios, 1(39), 66-85. https://doi.org/10.1016/j.it.2011.10.005

Freeman, R. E. (2004). The Stakeholder Approach Revisited. Zeitschrift Für Wirtschafts- 
Und Unternehmensethik, 5(3), 228-241. https://doi.org/10.3763/jsfi.2010.0008

Freeman, R. E. (2017). Five Challenges to Stakeholder Theory: A Report on Research in Progress (pp. 1-20). Stakeholder Management (Business and Society 360, Vol. 1). https://doi.org/10.1108/S2514-175920170000001

Gul, S. y Nouman, M. (2009). Innovation in Pakistan? SMES: Making the case for coupling model and looking beyond. Business \& Economic Review, 1(1), 20-25.

Hámori, B. y Szabó, K. (2011). Organizational innovations and their behavioral background. Scientific Management and Management Science Today. International Scientific Conference, University of Szeged, Faculty of Economics and Business, Szeged, Hungría. 4 y 5 de noviembre.

Han, J. K., Kim, N. y Srivastava, R. K. (1998). Market Orientation and Organizational Performance: Is Innovation a Missing Link? Journal of Marketing, 62(4), 30. https://doi.org/10.2307/1252285

He, Y., Gan, S. y Xiao, L. (2021). Can foreign suppliers act as "innovation springboards" for firms? Evidence from China. Research in International Business and Finance, 56. https://doi.org/10.1016/j.ribaf.2020.101353

Ibrahim, B. (2017). Access to Financial Institutions Financing as an Instrument of Growing Small Businesses in Nigeria: An Empirical Study. International Journal of Scientific and Research Publications, 7(10). www.ijsrp.org

Korhonen, H. M. E. y Kaarela, I. (2011). Corporate Customers' Resistance yo Industrial Service Innovations. International Journal of Innovation Management, 15(03), 479-503. https://doi.org/10.1142/s136391961100343x

Kraśnicka, T., Głód, W. y Wronka-Pośpiech, M. (2017). Management Innovation and Its Measurement. Journal of Entrepreneurship, Management and Innovation, 12(2), 95-121. https://doi.org/10.7341/20161225

Kruse, P. (2013). Customer Involvement in Organizational Innovation: Toward an Integration Concept. Proceedings of the Nineteenth Americas Conference on Information Systems, 2012, 1-10.

https://aisel.aisnet.org/cgi/viewcontent.cgi?referer=https://www.google.com/\&http sredir $=1 \&$ article $=1418 \&$ context $=$ amcis 2013

Lam, A. (2009). Organizational Innovation. In The Oxford Handbook of Innovation. Oxford University Press. https://doi.org/10.1093/oxfordhb/9780199286805.003.0005

Larios-Gomez, E. (2016). La Gestión de la Competitividad en la MIPYME Mexicana: Diagnóstico Empírico desde la Gestión del Conocimiento. Revista de 
Administração Da Unimep, 14(2), 177-209. https://doi.org/10.15600/16795350/rau.v14n2p177-209

Leal-rodriguez, A. L. y Albort-Morant, G. (2016). Linking Market Orientation, Innovation and Performance : an Empirical Study on Small Industrial Enterprises in Spain. Journal of Small Business Strategy, 26(1), 37-50. https://doi.org/10.13140/RG.2.1.2862.9523

Leovaridis, C., \& Popescu, G. (2015). Organizational Innovation - A Means to Enhance Quality of Life for Employees in Knowledge Economy. Dinámica de Gestión En La Economía Del Conocimiento, 3(1), 25-43.

Lima, L., Morais, R. y Mendes, F. (2017). The Influence of National Culture and Access to Innovation on Innovation Openness. XX SemeAd Seminários em Administração, 16. http://login.semead.com.br/20semead/arquivos/783.pdf

Lindblad, H. y Karrbom Gustavsson, T. (2021). Public clients ability to drive industry change: the case of implementing BIM. Construction Management and Economics, 39(1), 21-35. https://doi.org/10.1080/01446193.2020.1807032

Margir, A., Eiduzehi, N. T., Surizehi, Z. y Kamalipur, N. K. (2014). Studying the Relationship between Organizational Innovation and System thought in Service Organization. International Journal of Academic Research in Business and Social Sciences, 4(7), 25. https://doi.org/10.6007/ijarbss/v4-i7/984

Mitchell, R. K., Agle, B. R. y Wood, D. J. (1997). Toward a Theory of Stakeholder Identification and Salience: Defining the Principle of Who and What Really Counts. Academy of Management Review, 22(4), 853-886. https://doi.org/10.5465/AMR.1997.9711022105

Morrar, R., Gallouj, F. y Hammadou, H. (2013). Public-private innovation networks and innovation activities in French service firms. Journal of Innovation Economics, 10(2), 191. https://doi.org/10.3917/jie.010.0191

Mothe, C. y Nguyen-thi, T. U. (2011). Do firms rely on sources of information fo, $r$ organizational innovation? International Journal of Technology Management, 1/2(63), 125-144

Olson, P., Mazzarol, T., Volery, T., Clark, D. y Gough, N. (2011). A Multi-Country Strategic Alignment Study: Are SME Leaders' Decisions Aligned with their Country's Innovation Environment? 56th Annual ICSB World Conference, 60, 1-23. https://cemi.com.au/sites/all/publications/Olson, Mazzarol, Volery, Clark and Gough ICSB 2011.pdf 
Petiz Lousã, E. y Mendes Mónico, L. (2018). How can leadership and organizational culture predict innovation in small, medium and large enterprises? The Journal of Organizational Management Studies, 2018. DOI: 10.5171/2018. 703891

Racela, O. C. (2014). Customer Orientation, Innovation Competencies, and Firm Performance: A Proposed Conceptual Model. Procedia - Social and Behavioral Sciences, 148, 16-23. https://doi.org/10.1016/j.sbspro.2014.07.010

Ricciardi, F. (2014). Innovation Processes in Business Networks: Managing InterOrganizational Relationships for Innovational Excellence. Springer.

Rodríguez Valencia, N. E. y Olivares Contreras, R. A. (2019). Innovación de productos, de procesos y estratégica: un escenario de los empresarios futuros y actuales en México. RICEA Revista Iberoamericana de Contaduría, Economía y Administración, 8(16), 80-105. https://doi.org/10.23913/ricea.v8i16.135

Sahrom, N. S., Tan, C. L. y Yahya, S. (2016). Regulation, incentives and government policy: How does it stimulate R\&D engineers' innovative behaviours in malaysian biotechnology SMEs? Asian Academy of Management Journal, 21, 49-73. https://doi.org/10.21315/aamj2016.21.supp.1.3

Siguaw, J. A., Simpson, P. M. y Enz, C. A. (2006). Conceptualizing innovation orientation: A framework for study and integration of innovation research. Journal of Product Innovation Management, 23(6), 556-574. https://doi.org/10.1111/j.15405885.2006.00224.x

Silva, A. R. D., Ferreira, F. A. F., Carayannis, E. G. y Ferreira, J. J. M. (2021). Measuring SMEs ' Propensity for Open Innovation Using Cognitive Mapping and MCDA. IEEE Transactions on Engineering Management, 68(2), 396-407. https://doi.org/10.1109/TEM.2019.2895276

Steiber, A. y Alänge, S. (2015). Organizational innovation: a comprehensive model for catalyzing organizational development and change in a rapidly changing world. Triple Helix, 2(1), 0-25. https://doi.org/10.1186/s40604-015-0021-6

Tang, J., Pee, L. G. y lijima, J. (2013). Investigating the effects of business process orientation on organizational innovation performance. Information and Management, 50(8), 650-660. https://doi.org/10.1016/j.im.2013.07.002

Totterdill, P. (2015). Closing the Gap: The Fifth Element and Workplace Innovation. European Journal of Workplace Innovation, 1(1), 55-74. http://journal.uia.no/index.php/EJWI/article/viewFile/166/113

Townsend, W. (2010). Innovation and the value of failure. International Journal of 
Management and Marketing Research, 3(1), 75-84.

Van de Ven, A. H. (2016). Central Problems in the Management of Innovation. Management, 32(5), 590-607.

van de Vrande, V., de Jong, J. P. J., Vanhaverbeke, W. y de Rochemont, M. (2009). Open innovation in SMEs: Trends, motives and management challenges.

Technovation, 29(6-7), 423-437.

https://doi.org/10.1016/j.technovation.2008.10.001

Vega, L. A. (2016). Las nuevas formas de organización y la empresa socialmente responsable, piezas fundamentales para el desarrollo de la innovación. TEACs, $8(18), 31-45$.

Voica, O. M., Stancu, S. y Naghi, L. E. (2016). Stimulating the innovative proposals from employees. Ecoforum, 5(1), 235-240.

Watson, R., Wilson, H. N., Smart, P. y Macdonald, E. K. (2018). Harnessing Difference: A Capability-Based Framework for Stakeholder Engagement in Environmental Innovation. Journal of Product Innovation Management, 35(2), 254-279. https://doi.org/10.1111/jpim.12394

Zhao, Q., Tsai, P. H. y Wang, J. L. (2019). Improving financial service innovation strategies for enhancing China's banking industry competitive advantage during the fintech revolution: A hybrid MCDM model. Sustainability (Switzerland), 11(5), 1-29. https://doi.org/10.3390/su11051419

Zhuang, Y. (2005). Does Electronic Business Create Value for Firms? an Organizational Innovation Perspective. Journal of Electronic Commerce Research, 6, 146151,153-156. http://ojs.jecr.org/jecr/sites/default/files/06_2_p05.pdf

\section{Otros docuementos consultados}

Instituto Nacional de Estadísitca y Geografía - INEGI (2017). Encuesta sobre Investigación y Desarrollo de Tecnología 2017 ESIDET. Síntesis metodológica (Documentos Meteodológico).

https://www.inegi.org.mx/contenido/productos/prod_serv/contenidos/espanol/bvi negi/productos/nueva_estruc/702825109073.pdf

Instituto Nacional de Estadísitca y Geografía - INEGI (2019). INEGI Presenta resultados de la encuesta nacional sobre productividad y competitividad de las micro, pequeñas y medianas empresas (ENAPROCE) 2018. Comunicado de prensa 
(448/19).

https://www.inegi.org.mx/contenidos/saladeprensa/boletines/2019/especiales/EN APROCE2018.pdf

Organización para la Cooperación y el Desarrollo Económicos - OCDE. (2019). OECD SME and Entrepreneurship Outlook 2019. In OECD SME and Entrepreneurship Outlook 2019. https://doi.org/10.1787/34907e9c-en

Organización para la Cooperación y el Desarrollo Económicos -OCDE y la Comunidad Europea UE. (2018). Oslo Manual. https://doi.org/10.1787/9789264304604-en

Organización Internacional del Trabajo - OIT. (2020). Entorno MIPYME: Medidas de apoyo a la micro, pequeña y mediana empresa en América Latina y el Caribe frente a la crisis de la COVID-19. https://www.ilo.org/wcmsp5/groups/public/--ed_dialogue/---act_emp/documents/publication/wcms_762907.pdf 